\title{
Synthesis and Characterization of $\mathrm{SnO}_{2} \mathrm{Nano}$ Particles for Carbon Absorbing Applications
}

\author{
V. RATCHAGAR and K. JAGANNATHAN* \\ Department of Physics, SRM University, City Campus, \\ Vadapalani, Chennai-600026, India. \\ *Corresponding author E-mail: kjagan81@gmail.com \\ http://dx.doi.org/10.13005/ojc/320121
}

(Received: October 05, 2015; Accepted: February 27, 2016)

ABSTRACT

The $\mathrm{SnO}_{2}$ nanoparticles was synthesized by Microwave assisted technique.From the powder XRD the particle size was calculated using Scherrer formula, it from $57 \mathrm{~nm}$. The absorption spectrum was recorded from $1100 \mathrm{~nm}$ to $190 \mathrm{~nm}$, and the optical band gap was calculated using Touc plot. The band gap value $3.16 \mathrm{eV}$. The functional groups were confirmed by FTIR spectrum. The FE-SEM analysis reveals the morphology of $\mathrm{SnO}_{2}$ nano particles. The synthesized $\mathrm{SnO}_{2}$ nano particles were used as a catalyst to reduce carbon from automobiles fume. The detailed study on carbon emission was reported.

Key words: Nano materials, $\mathrm{SnO}_{2}$, SEM, Carbon Emission.

\section{INTRODUCTION}

In recent years the researcher's interests have been involved in on low dimensional inorganic semiconducting metal oxide nanomaterials owing to their unique optical, chemical, and electrical properties. As these properties were deeply influenced by the size and morphology of the nanostructures, the preparation of nano sized crystallites with different morphologies provided an opportunity to explore the possible changes in their physical and chemical properties with size and shape ${ }^{1,2,3}$. Now days the researchers were involved to prepare the one-dimensional nonmaterials to increasing their numerous applications ${ }^{4}$. The tinoxide $\left(\mathrm{SnO}_{2}\right)$ is the one of the interesting candidates due to its potential applications like gas sensors ${ }^{5,6}$, solarcells ${ }^{7}$, lithium batteries ${ }^{8}$, and in transparent conductive electrodes ${ }^{9}$. In recent days, nanowire and nanotube based materials have been well demonstrated as building blocks for nano circuits, nano systems and nano-optoelectronics. For the same reasons, researchers focuses their interest on one-dimensional nano structured $\mathrm{SnO}_{2}$ materials ${ }^{10-13}$.

In quantum size effect, when the particle size of a semiconductor is compared to the Bohr 
radius $^{14}$, the ratio of surface atomsto those with the interior enhances the surface properties of the materials. Due to these effects, band gap of the semiconductor nano particles has been modified, which makes a lot of changes in their electronic properties when compared to the bulk. Thus, there is an increase in the band gap i.e. the visible spectra show a blue shift, which is assigned to quantum size effect ${ }^{15}$. This stimulated a great interest in both basic and applied research in semiconductor oxide nano materials. Many methods have been applied by researchers to synthesize the $\mathrm{SnO}_{2}$ nano materials ${ }^{16}$ like sol-gel route, thermal decomposition ${ }^{17}$, coprecipitation $^{18}$, microwave-assisted solution ${ }^{19}$, gas phase condensation ${ }^{20}$ and laser ablation ${ }^{21}$ etc.

In this paper, we have reported the $\mathrm{SnO}_{2}$ nanoparticles as catalyst to reduce carbon from automobile engine. The synthesized $\mathrm{SnO}_{2}$ material was characterized using various techniques like XRD, UV, FTIR, FE-SEM and EDX.

\section{MATERIALS AND METHODS}

The $0.1 \mathrm{M}$ solution of Tin (II) chloride was prepared using deionized water as solvent. The $\mathrm{pH}$ solution was maintained by liquid ammonia. The resulting precipitate was washed using deionized water, until no chlorine ions are detected (silver nitrate test). Further to remove $\mathrm{NH}^{4+}$ ions the precipitate was washed using ethanol. Then it was irradiated with house hold microwave oven for 10 min. The radiation frequency was $2.45 \mathrm{GHz}$ and its power up to $1 \mathrm{KW}$. The find product gives $\mathrm{SnO}_{2}$ nanoparticles.

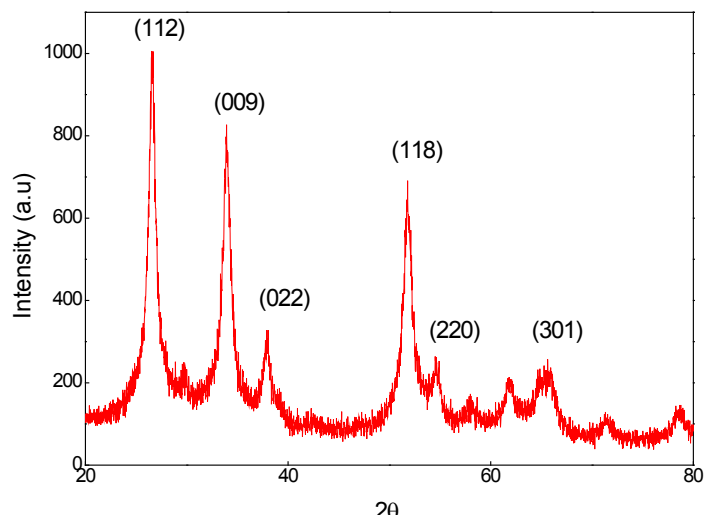

Fig. 1: XRD pattern $\mathrm{SnO}_{2}$ nano particles

\section{Characterization} XRD analysis

The fig. 1 shows powder XRD pattern of $\mathrm{SnO}_{2}$ nanoparticles shown in fig.1.The powder sample was scanned in steps of $0.02{ }^{\circ}$ for time interval of 10 s over a $2 \theta$ range of $20-80$ in Xport high score. The observed pattern has prominent peaks at (112), (009), (022), (118), (220) and (301), are well coincide with JCPDS file no 41- 1445are confirms the formation of $\mathrm{SnO}_{2}$ nano particles. The $\mathrm{SnO}_{2}$ shows tetragonalstructure. The sharpness of peaks shows that $\mathrm{SnO}_{2}$ nanoparticles are highly crystalline. The particle size of the as-prepared powder was calculated using Debye Scherrer formula, $, 2,32$

$$
\mathrm{D}=\mathrm{k} \lambda / \beta \cos
$$

where $D$ is the particle size, $\lambda$ is the $X$-ray wavelength, $\beta$ is the full width at half maximum of the diffraction peak, and $\theta$ is the Bragg diffraction angle of corresponding peaks. The average particle size was found to be $56 \mathrm{~nm}$.

\section{UV-vis absorption spectrum}

The fig. 2 shows that UV-visible absorption spectrum of $\mathrm{SnO}_{2}$ nano particles. The UV spectrum carried from 190 -1100 nm. UV-visible spectroscopy provides useful information about the optical band gap of the semiconductors. For the semiconductor nanoparticles, the quantum confinement effect is expected and the absorption edge will be shifted to a higher energy when the particle size decreases ${ }^{22,23}$. In the absorption spectrum shows the cut of wavelength of $\mathrm{SnO}_{2}$ nano particles is $210 \mathrm{~nm}$.

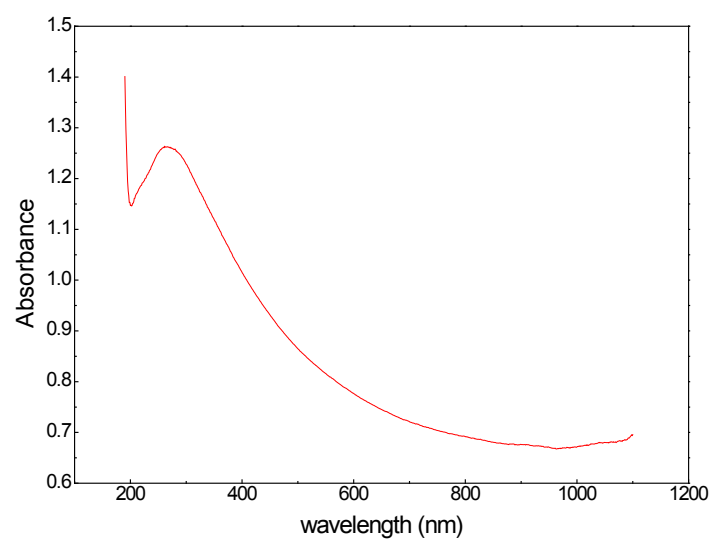

Fig. 2: UV absorption spectrum of $\mathrm{SnO}_{2}$ nano particles 


\section{Calculation of optical band gap}

The fig. 2 shows the optical bandgap of various $\mathrm{pH}$ samples were determined from the absorption spectra. The relation between the optical absorption coefficient hí and the photon energy was given by Mott and Davis ${ }^{24}$ as

$$
\alpha(h v)=B\left(h v-E_{g}\right)^{n} / h v
$$

where $B$ is a constant dependent on the transition probability, $\mathrm{h}$ is Planck's constant, í is the frequency of the radiation, and $E_{g}$ is the optical energy gap. The type of transition responsiblefor the absorption depends on the value of $n$ an index that can take any of the values $1 / 2,3 / 2,2$ or 3 for direct-allowed, direct-forbidden, indirect-allowed, and indirect forbidden transitions, respectively. In the present case, $n=1 / 2$, which means an allowed direct transition. The optical absorption coefficient was calculated from the absorbance a using the following equation ${ }^{25}$

$$
\alpha(v)=2.303 \mathrm{~A} / \mathrm{d}
$$

Where $d$ is the thickness of the sample in centimetre and $A$ is calculated using the formula

$$
A=\ln \left(I_{0} / I_{t}\right)
$$

Where $\mathrm{I}_{0}$ andl $\mathrm{l}_{\mathrm{t}}$ are the intensities of incident and transmitted light, respectively.

For the determination of the direct optical band gap $a^{2}$ was plotted as function of photon energy $h \gamma$. When the dimensions of nanoparticles approach the exciton Bohr radius, a blue shift in energy is observed due to the quantum confinement. The band gap of $\mathrm{SnO}_{2}$ was found to be $3.16 \mathrm{eV}$. The effective mass model [26] is commonly used to study the size dependence of optical properties of quantum systems. The resultant values of $E_{g}$ for freshly prepared $\mathrm{SnO}_{2}$ nanoparticle is found to be about $3.16 \mathrm{eV}$ with splitting of energy ${ }^{2,27}$.

\section{FT-IR spectrum}

The FT-IR spectrum was recorded for $\mathrm{SnO}_{2}$ nano particles after microwave treatment (fig. 4). The FTIR spectrum is recorded using Avatar 330 FT-IR thermo nicolet spectrometer in the wavelength range 400-4000 $\mathrm{cm}^{-1}$ by $\mathrm{KBr}$ pellet technique. The absorption band at $539 \mathrm{~cm}^{\prime 1}$ was ascribed to the terminal oxygen vibration $\left(i_{\mathrm{Sn}-\mathrm{OH}}\right)^{29}$ for the dried precipitate. It was obvious that the hydroxyl group changed into oxide group during microwave radiation. After heating the product at higher sintering temperatures $(1 \mathrm{KW})$ a new broad band centred at $620 \mathrm{~cm}^{\prime 1}$ appeared which was characteristic of oxide-bridge functional group $\left(i_{\text {OSno }}\right)^{30}$.It was obvious that at this temperature the $\mathrm{SnO}_{2}$ had changed into SnO.The peaks at $1631 \mathrm{~cm}{ }^{\prime 1}$ were assigned to $\mathrm{NH}$ deformation ofammonia and $\mathrm{NH}$ stretching vibration respectively. The absorption band at $3323 \mathrm{~cm}$ "1was mainly due toí ${ }_{\mathrm{OH}}$ stretching vibration of surface hydroxyl group oradsorbed water. For low $\mathrm{pH}$ value $\mathrm{OH}$ groups were still present at the surface and at the interior of the $\mathrm{SnO}$ particles. The remaining low signals attributed to some $\mathrm{OH}$ groups at the surface

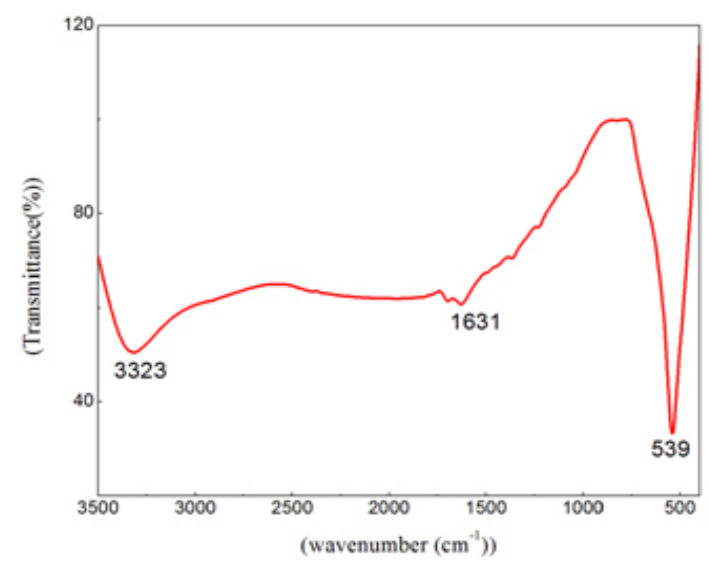

Fig. 4: FT-IR spectrum of $\mathrm{SnO}_{2}$ Nano particles

Fig. 3: Optical band gap of $\mathrm{SnO}_{2}$ Nano particles 
were probably due to the re-absorption of water from the ambientatmosphere ${ }^{31}$.

\section{Scanning Electron Microscopy (SEM)}

The morphology and chemical composition of the microwave synthesized tin oxide nanostructures were analyzed by SEM (fig.5). The micrograph of $\mathrm{SnO}_{2}$ sample shows the typical morphology of the $\mathrm{SnO}_{2}$ powders synthesized,
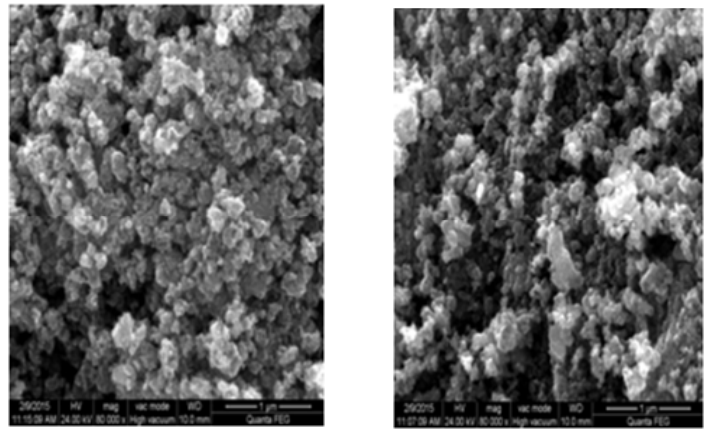

Fig. 5: SEM image of $\mathrm{SnO}_{2}$ Nano particles characterized by the presence of particles with an average size of about $1 \mu \mathrm{m}$ or less. In this study $\mathrm{SnO}_{2}$ nanoparticles found to be spherical.

\section{EDX spectrum}

The fig 6 shows the EDX spectrum of $\mathrm{SnO}_{2}$ nano particles. The spectrum is confirmed the presence of elemental (stannum) tin oxide and oxygen. There was no other element not observed in EDX spectra. It shows the synthesized $\mathrm{SnO}_{2}$ is in high pure form.

\section{Measurements of $\mathrm{CO}_{2}$ level}

The synthesized $\mathrm{SnO}_{2}$ nanoparticles were used as catalyst to reduce the carbon content of automobile fume. For this measurement we have chosen the pulsar bike (2002 model). The emission test was carried out by conventional emission test instrument, it records the emission for 0-60 seconds and it is shown in fig. 7a. Then the synthesized catalyst was spread on the wool rolled and put in the cylindrical tube. The tube fixed in the outer core

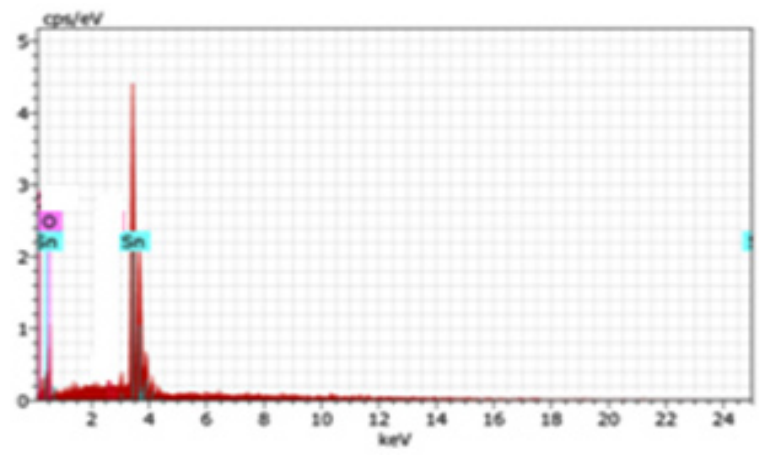

Fig. 6: EDX image of $\mathrm{SnO}_{2}$ Nano particles

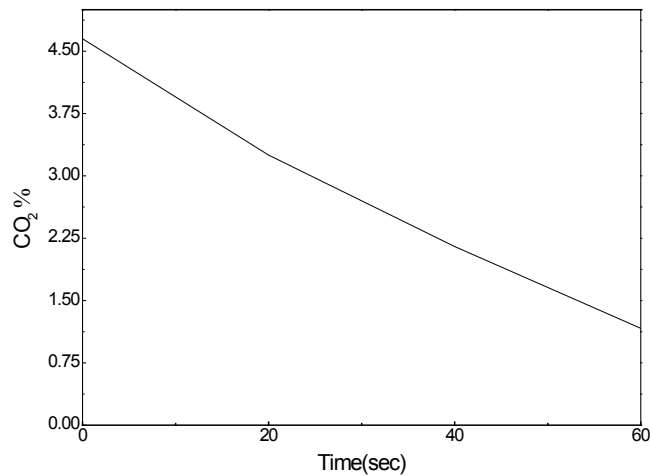

a

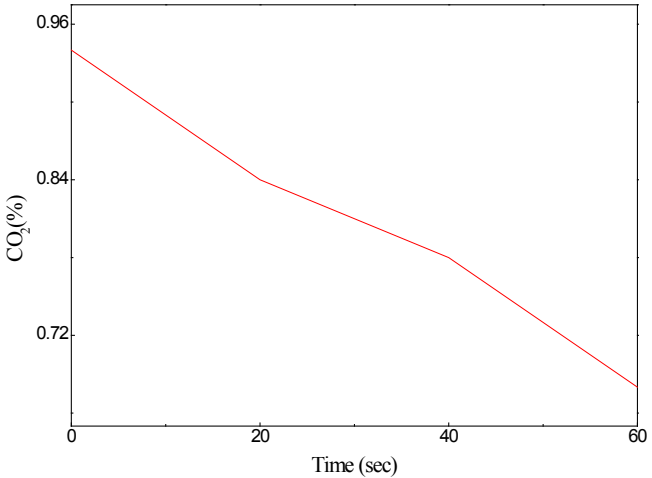

b

Fig. 7: (a) without catalyst (b) with catalyst 
of the silencer. The bike get started the smoke was measured the $\mathrm{CO}_{2}$ gas during the interval of the 0-60 secondsand the results were shown in fig. $7 \mathrm{~b}$. The amount of emitted $\mathrm{CO}_{2}$ is found reduced while using $\mathrm{SnO}_{2}$ catalyst. In this way the $\mathrm{SnO}_{2}$ catalyst used as a converter used redox reactor to reduce the pollutants of the two wheelers.

\section{CONCLUSION}

We have successfully synthesized $\mathrm{SnO}_{2}$ nano particles by microwave assisted method. The powder $X$-ray diffraction shows the good crystalline nature and particle size calculated usingDebye scherrer formula. The calculated particle $57 \mathrm{~nm}$. The band gap of $\mathrm{SnO}_{2}$ nano particles is measured from Tauc relation. Morphology was investigated carried from SEM. The EDX spectrum gives atomic percentage level of $\mathrm{SnO}_{2}$ nano particles. The $\mathrm{SnO}_{2}$ is identified as suitable material to reduce the Carbon from automobiles fume.

\section{ACKNOWLEDGMENTS}

The authors convey the heart full gratitude to the management of SRM university for their motivation and support.

\section{REFERENCES}

1. Das, S.;Kar,S.; Chaudhuri, S.; J. Appl. Phys2006, 99, 114303

2. Gudiksen, M.S,;Lauhon,L. J.; Wang,J.; Smith,D.; Lieber,C. M.;Nature London2002, 415, 617-620.

3. Göpel, W.; Schierbaum,K. D.; Sens. Actuators $B, 1995,1,26-27$

4. Zhang,G.; Liu,M.;Sens. Actuators B, 2000, 69, 144-152.

5. Ansari,G.;Boroojerdian,D.;Sainker, S.R.;Karekar,R. N.;Aiyer,R. C.; Kulkarni,S. K.; Thin Solid Films, 1997, 295(1)271-276.

6. Law, M.; Kind, H.; Messer, B.; Kim, F.; Yang, P.; Angew.D.; Chem.,Int. Ed.2002, 41, 24052408.

7. Harrison, P. G.; Willet, M.; J.Nature1988, 332337.

8. Idota, Y.; Kubota, T.; Matsufuji, A.; Maekawa, Y.; Miyasaka, T.Science, 1997, 276, 13951397.

9. He, Y. S.; Campbell, J. C.; Murphy, R. C.; Arendt, M. F.; Swinnea, J. S.; J. Mater. Research.1993,8,3131.

10. Wang, Y.; Jiang, X.; Xia, Y.; J. Am. Chem. Soc,2003, 125, 16176-16177

11. Kolmakov, A.; Zhang, Y.; Cheng, G.; Moskovits, M.AdV. Mater,2003, 15,997-1000.

12. Law, M.; Kind, H.; Messer, B.; Kim, F.;Yang, P.; Angew. Chem., Int. Ed,2002,41,2405-2408.

13. Comini, E.; Faglia, G.; Sberveglieri, G.; Pan, Z. W.;Wang, Z.; J. Appl. Phys.Lett.,2002,81,18691871.

14. FengGu, Shu Fen Wang, Chun Feng Song,
Meng Kai Lu, Yong Xin Qi, Guang Jun Zhou, Dong Xu, Duo Rong Yuan, Chemical Physics Letters,2003,372,451-454.

15. FengGu, Shu Fen Wang, Meng Kai Lu, Guang Jun Zhou, Dong Xu and Duo Rong Yuan, J. Phys. Chem. B,2004, 108,81198123

16. Ying,Z.; Wan,Q.; Song,Z.T.; Feng, S.L.; Mater. Lett, 2005,59,1670-1672.

17. Liu,Y.;Dong, J.;Liu, M.; Adv.Mater.2004, 16,353356.

18. Ki Chang Song, Yong Kang, Materials Letters, 2000,42,283-289.

19. Hallila,H.; Méninia, P.;Auberta,H.;Procedia Chemistry,2009, 1,935.

20. Zhang,J.;Gao,L.; Chem. Lett,2003,32,458.

21. Yang,H.; Song,X.; Zhang,X.; Ao,W.; Qui,G.;Mater. Lett,2003,57, 3124-3127.

22. Moldovan,D.; Yamakov,V.; Wolf,D.; Phillport,S.R.;Phys. Rev. Lett.2002,89,206101206104

23. Das,S.; Kar, S.;Choudhary,S. ; J. Appl. Phys.2006,99,114303-114309.

24. Mott,N.F.; Davis,E.A.;Electronic Processes in Non-Crystalline Materials, Oxford, Clarendon, 1979.

25. Fox,M.; Optical Properties of Solids, Oxford University Press, New York, 2001.

26. Pankov,Jl.;Optical Processes in Semiconductors, Prentice-Hall Inc.,EnglewoordCliffs, NJ; 1971.

27. Patil G.E., Kajale D.D., Gaikwad V.B., Jain G.H., International Nano Letters 2012, 2:17. 
28. Naje A.N., Norry. A.S., Suhail A.M., International Journal of Innovative Research in Science, Engineering and Technology2013,2 (12), 7068-7072.

29. Zhang Jianrong, GaoLian, Inorganic Chemistry Communications, 2004, 7,91-93.
30. RaiRadheshyam, T.D.;Senguttuvan, S.T.; Lakshmikumar, Computational MaterialsScience, 2006, 37, 15-19.

31. Yung-Jen Lin, Ching-Jiunn Wu, Surface and Coating Technology, 1996, 88,239-247 